\title{
SCN5A mutations and polymorphisms in patients with ventricular fibrillation during acute myocardial infarction
}

\author{
TIM BOEHRINGER ${ }^{1,2}$, PETER BUGERT ${ }^{1,2}$, MARTIN BORGGREFE $^{2,3}$ and ELIF ELMAS ${ }^{2,3}$ \\ ${ }^{1}$ Institute of Transfusion Medicine and Immunology, German Red Cross Blood Service Baden-Württemberg, Hessen 68167; \\ ${ }^{2}$ Medical Faculty Mannheim, Heidelberg University; ${ }^{3}$ First Department of Medicine, \\ University Medical Centre Mannheim, Heidelberg University, Mannheim 68167, Germany
}

Received September 11, 2013; Accepted April 1, 2014

DOI: $10.3892 / \mathrm{mmr} .2014 .2401$

\begin{abstract}
Mutations in the $S C N 5 \mathrm{~A}$ gene encoding the $\mathrm{Na}_{\mathrm{v}} 1.5$ channel $\alpha$-subunit are known to be risk factors of arrhythmia, including Brugada Syndrome and Long QT syndrome subtype 3 . The present study focused on the role of SCN5A variants in the development of ventricular fibrillation (VF) during acute myocardial infarction (AMI). Since VF during AMI is the major cause of sudden death in the Western world, SCN5A mutations represent genetic risk factors for sudden death. By exon re-sequencing, the entire coding region and flanking intron regions were sequenced in $46 \mathrm{AMI} / \mathrm{VF}^{+}$ patients. In total, nine single nucleotide variants were identified of which four represented common single nucleotide polymorphisms (SNPs; 87G $>$ A, 1673A $>$ G, IVS16-6C $>$ T and $5457 \mathrm{~T}>\mathrm{A})$. Only five rare variants were identified, each in only one patient. Only two of the rare variants represented missense mutations $(3578 \mathrm{G}>\mathrm{A}$ and $4786 \mathrm{~T}>\mathrm{A})$ ). The common SNPs and the missense mutations were also genotyped using polymerase chain reaction methods in $79 \mathrm{AMI} / \mathrm{VF}^{-}$patients and 480 healthy controls. The SNPs did not demonstrate significant differences in allele and genotype frequencies between the study groups. The $3578 \mathrm{G}>\mathrm{A}$ mutation was identified in one out of the 480 controls, whereas the $4786 \mathrm{~T}>\mathrm{A}$ mutation was not present in $\mathrm{AMI} / \mathrm{VF}^{-}$patients and controls. In conclusion, the majority of $\mathrm{AMI} / \mathrm{VF}^{+}$patients demonstrated a wild type sequence or common SNPs in SCN5A. Only two out of $46(4.3 \%) \mathrm{AMI} / \mathrm{VF}^{+}$patients revealed mutations that may be involved in Nav1.5 dysfunction and VF. However, this requires further functional validation.
\end{abstract}

Correspondence to: Professor Elif Elmas, First Department of Medicine, University Medical Centre Mannheim, Heidelberg University, 1-3 Theodor-Kutzer-Ufer, Mannheim 68167, Germany E-mail: elif.elmas@umm.de

Key words: genetic risk factor, SCN5A gene variant, exon resequencing, myocardial infarction, ventricular fibrillation, arrhythmia

\section{Introduction}

In the Western world ventricular fibrillation (VF) during acute myocardial infarction (AMI) is the major cause of sudden cardiac death (SCD) (1). VF occurs in $10 \%$ of cases within the first hours following the symptoms of an AMI $(2,3)$. In addition to established risk factors for VF, a significant genetic component may be detected using extensive observational population studies (4-7). In primary electrical heart diseases, including Long QT syndrome (LQTS), Short QT syndrome and Brugada syndrome, genes encoding ion channels are mutated.

The SCN5A gene encodes the major cardiac voltage-gated sodium channel $\alpha$-subunit $\mathrm{Na}_{\mathrm{v}} 1.5$ and is located on chromosome 3p21-24 (8). Exons 2-28 contain the protein-coding sequence and several splice variants have been described (9). The major role of the sodium channel is the rapid depolarization at the beginning of an action potential and the transmission of electrical impulses in the heart myocardia $(8,10)$. Mutations in SCN5A may affect different mechanisms, including channel activation, inactivation and reactivation or may lead to complete loss of function (11). Mutations were identified in different types of arrhythmias (12-15), i.e. in 10-20\% of patients with Brugada syndrome and in $6 \%$ of patients with Long QT syndrome subtype 3 (LQTS3). Common polymorphisms in SCN5A modulate the biophysical defects of SCN5A mutations (16-19) or may be associated with an increased risk of SCD (20). The potential association of SCN5A variants with VF during AMI remains unclear. Among a small cohort of 19 patients suffering from VF during AMI, only one demonstrated a missense mutation in SCN5A (21). In our previous study on 240 AMI patients, including 73 patients with primary VF an association of the $S C N 5 A-H 558 \mathrm{R}$ polymorphism with the risk of VF or AMI was not identified (22).

The present study aimed to investigate the role of SCN5A mutations and polymorphisms in the development of VF during AMI. Screening for DNA sequence variation in the coding region of $S C N 5 A$ was performed by exon re-sequencing in patients suffering from VF during AMI $\left(\mathrm{AMI} / \mathrm{VF}^{+}\right)$. For common single nucleotide polymorphisms (SNPs) and rare mutations identified in the $\mathrm{AMI} / \mathrm{VF}^{+}$patients polymerase chain reaction with sequence-specific primers (PCR-SSP) was developed. In order to estimate the role of these gene variants 
Table I. Baseline characteristics of patients and controls.

\begin{tabular}{|c|c|c|c|c|}
\hline Characteristic & $\begin{array}{c}\mathrm{AMI} / \mathrm{VF}^{+} \\
\text {patients }(\mathrm{n}=49)\end{array}$ & $\begin{array}{c}\mathrm{AMI} / \mathrm{VF}^{-} \\
\text {patients }(\mathrm{n}=74)\end{array}$ & $\begin{array}{l}\text { Controls } \\
(n=480)\end{array}$ & $\begin{array}{l}\text { Significance } \\
(\mathrm{P}-\mathrm{value})^{\mathrm{a}}\end{array}$ \\
\hline No. of males (\%) & $44(91.8)$ & $53(71.6)$ & $288(60.0)$ & $\begin{array}{l}0.045^{\mathrm{a}} \\
<0.001^{\mathrm{b}}\end{array}$ \\
\hline Age $($ mean $\pm \mathrm{SD})$ & $60.7 \pm 10.0$ & $61.0 \pm 12.0$ & $57.3 \pm 6.7$ & $\begin{array}{l}0.903^{\mathrm{a}} \\
0.001^{\mathrm{b}}\end{array}$ \\
\hline \multicolumn{5}{|l|}{ CAD, n (\%) } \\
\hline 1-vessel disease & $28(57.1)$ & $27(36.5)$ & - & $0.028^{\mathrm{a}}$ \\
\hline 2-vessel disease & $18(36.7)$ & $22(29.7)$ & - & $0.438^{\mathrm{a}}$ \\
\hline 3-vessel disease & $3(6.1)$ & $25(33.8)$ & - & $<0.001^{\mathrm{a}}$ \\
\hline No. of patients with hypertension (\%) & $27(55.1)$ & $40(54.1)$ & - & $0.909^{\mathrm{a}}$ \\
\hline No. of patients with hyperlipoproteinemia (\%) & $26(53.1)$ & $47(63.5)$ & - & $0.266^{\mathrm{a}}$ \\
\hline No. of smokers (\%) & $26(53.1)$ & $38(51.4)$ & - & $0.853^{\mathrm{a}}$ \\
\hline No. of patients with diabetes mellitus (\%) & $11(22.4)$ & $17(23.0)$ & - & $0.946^{\mathrm{a}}$ \\
\hline $\begin{array}{l}\text { No. of patients with a family history of } \\
\text { myocardial infarction }(\%)\end{array}$ & $12(24.5)$ & $17(23.0)$ & - & $0.830^{\mathrm{a}}$ \\
\hline
\end{tabular}

${ }^{\mathrm{a}} \mathrm{AMI} / \mathrm{VF}^{+}$vs. AMI/VF; ${ }^{-}{ }^{\mathrm{b}} \mathrm{AMI}$ vs. controls. AMI, acute myocardial infarction; VF, ventricular fibrillation; CAD, coronary artery disease; $\mathrm{SD}$, standard deviation.

as risk factors for VF or AMI, the present study additionally genotyped $\mathrm{AMI} / \mathrm{VF}^{-}$patients and healthy controls.

\section{Materials and methods}

Patients and controls. Patients were recruited from the First Department of Medicine (Cardiology), University Medical Centre (Mannheim, Germany). The controls were recruited at the Institute of Transfusion Medicine and Immunology, German Red Cross Blood Service (Mannheim, Germany). In total 49 Patients who suffered from VF during AMI $\left(\mathrm{AMI} / \mathrm{VF}^{+}\right.$) and $74 \mathrm{AMI}$ patients without VF (AMI/VF-) were included. Additionally, a control group of 480 healthy blood donors was analyzed. The baseline characteristics of the study individuals are summarized in Table I. All patients and controls provided written consent to use biological material for molecular genetic research purposes. The study was approved by the ethics committee of the Medical Faculty Mannheim, Heidelberg University (Mannheim, Germany). DNA was extracted from EDTA blood samples of all study individuals using commercial kits (QIAamp ${ }^{\circledR}$ DNA Blood Mini kit; Qiagen, Hilden, Germany).

Mutation screening and genotyping. SCN5A exons 2-28 were amplified from genomic DNA using flanking intron primers (Table II). Sequencing was performed on the two strands with the use of the amplification primers.

For the single nucleotide variations (mutations and SNPs) identified by mutation screening PCR-SSP methods were established according to standard protocols (23) and the primers are provided in Table III. In addition, PCR-SSP methods were developed for $S C N 5 A$ variants $1062 \mathrm{~T}>\mathrm{C}, 354 \mathrm{G}>\mathrm{C}, 287 \mathrm{C}>\mathrm{T}$ and $1199 \mathrm{G}>\mathrm{C}$, which were associated with arrhythmia in previous studies $(21,24)$. All patients and controls were genotyped for the SCN5A variants using PCR-SSP.

Statistical analysis. Fisher exact and $\chi^{2}$ tests were performed to investigate differences between the study cohorts in the baseline characteristics as well as allele and genotype frequencies of the SCN5A polymorphisms. SPSS statistical software version 12.0 (SPSS, Inc., Chicago, IL, USA) was used for the statistical analysis.

\section{Results and Discussion}

Screening for $S C N 5 A$ sequence variations in the entire coding region and flanking intron regions was achieved by exon re-sequencing in $46 \mathrm{AMI} / \mathrm{VF}^{+}$patients. In total, nine single nucleotide variations were identified and were all listed in the Single Nucleotide Polymorphism Database and LQTS gene Leiden Open Variation Database (25) (Table IV). Four variants $(87 \mathrm{G}>\mathrm{A}, 1673 \mathrm{~A}>\mathrm{G}, \mathrm{IVS} 16-6 \mathrm{C}>\mathrm{T}$ and $5457 \mathrm{~T}>\mathrm{A})$ represented common SNPs of which only $1673 \mathrm{~A}>\mathrm{G}$ (His558Arg) was a missense variant. However, all SNPs have previously been described as normal variants without an association with arrhythmia (26-31).

The $630 \mathrm{G}>\mathrm{A}$ variant is rare and was found in exon $6 \mathrm{~b}$ of one $\mathrm{AMI} / \mathrm{VF}^{+}$patient. The amino acids encoded by exon $6 \mathrm{~b}$ are present in the major 'adult' SCN5A isoform, whereas exon $6 \mathrm{a}$ is alternatively spliced in the 'neonatal' isoform (9). However, the nucleotide change $630 \mathrm{G}>\mathrm{A}$ does not alter the encoded amino acid and no effect on protein function was assumed. The variants $3183 \mathrm{G}>\mathrm{A}$ and $4509 \mathrm{C}>\mathrm{T}$ also represent rare and silent variants, each found in only one $\mathrm{AMI} / \mathrm{VF}^{+}$patient. The rare and silent variants were not further investigated in the present study. 
Table II. Primers for amplification and re-sequencing of SCN5A exons 2-28.

\begin{tabular}{|c|c|c|c|c|c|}
\hline Target & Binding region & Direction & Sequence $\left(5^{\prime}-3^{\prime}\right)$ & $\begin{array}{l}\text { Final } \mathrm{MgCl}_{2} \\
\text { conc. }(\mathrm{mM})\end{array}$ & Size (bp) \\
\hline \multirow{2}{*}{$\begin{array}{l}\text { Exon } 2 \\
(\text { part I) }\end{array}$} & Intron 1 & Sense & ctgtccetgggcatagaatc & 3.5 & 188 \\
\hline & Exon 2 & Antisense & ttctctgccatgcgettctc & & \\
\hline \multirow{2}{*}{$\begin{array}{l}\text { Exon } 2 \\
\text { (part II) }\end{array}$} & Exon 2 & Sense & cagcttccgcaggttcacac & 2.0 & 288 \\
\hline & Intron 2 & Antisense & ggagttgcacagaagggtag & & \\
\hline \multirow[t]{2}{*}{ Exon 3} & Intron 2 & Sense & tctacacaagggcctaatgctac & 2.0 & 274 \\
\hline & Intron 3 & Antisense & agggaatcagcgctactctc & & \\
\hline \multirow[t]{2}{*}{ Exon 4} & Intron 3 & Sense & cccatgctgctcagctttcc & 2.0 & 152 \\
\hline & Intron 4 & Antisense & gtggagaagaggccetgaag & & \\
\hline \multirow[t]{2}{*}{ Exon 5} & Intron 4 & Sense & acgtaaggaacctggagaacc & 3.5 & 306 \\
\hline & Intron 5 & Antisense & agggaggaagccagaaagag & & \\
\hline \multirow[t]{2}{*}{ Exon 6} & Intron 5 & Sense & caggcggtggttctgctttg & 2.0 & 424 \\
\hline & Intron 6 & Antisense & aaggcccaggcatatccetc & & \\
\hline \multirow[t]{2}{*}{ Exon 7} & Intron 6 & Sense & cgtgcttgttcttgccttcc & 3.5 & 299 \\
\hline & Intron 7 & Antisense & gctggtctcacaaagtcttc & & \\
\hline \multirow[t]{2}{*}{ Exon 8} & Intron 7 & Sense & cctggatgcaaggcggaaac & 2.0 & 274 \\
\hline & Intron 8 & Antisense & gaagggtcctggcaggtaag & & \\
\hline \multirow[t]{2}{*}{ Exon 9} & Intron 8 & Sense & tggcactaggtttgtgaagc & 2.0 & 325 \\
\hline & Intron 9 & Antisense & ctgagcccacacttgctgtc & & \\
\hline \multirow[t]{2}{*}{ Exon 10} & Intron 9 & Sense & cctggcacaactagactagg & 3.5 & 332 \\
\hline & Intron 10 & Antisense & agtcaggtgagggcttagag & & \\
\hline \multirow[t]{2}{*}{ Exon 11} & Intron 10 & Sense & ggctgcacaaagtctcaatg & 2.0 & 348 \\
\hline & Intron 11 & Antisense & aaacaggaagcgcagagatg & & \\
\hline \multirow[t]{2}{*}{ Exon 12} & Intron 11 & Sense & ccctctcctcatgccettag & 2.0 & 425 \\
\hline & Intron 12 & Antisense & tgctgtggtgcctgcatctc & & \\
\hline \multirow[t]{2}{*}{ Exon 13} & Intron 12 & Sense & cccaggctgacgcaaatctc & 2.0 & 242 \\
\hline & Intron 13 & Antisense & tgggtcaggctgggataaag & & \\
\hline \multirow[t]{2}{*}{ Exon 14} & Intron 13 & Sense & gtcatctcccagagcaagtc & 2.0 & 379 \\
\hline & Intron 14 & Antisense & caggatgcccatttgagagc & & \\
\hline \multirow[t]{2}{*}{ Exon 15} & Intron 14 & Sense & ggccagggagtctttccatc & 2.0 & 283 \\
\hline & Intron 15 & Antisense & ttgggttgtgccgagccttc & & \\
\hline \multirow[t]{2}{*}{ Exon 16} & Intron 15 & Sense & ccagagcccttcacaaggtc & 2.0 & 442 \\
\hline & Intron 16 & Antisense & gctgggtagatgagtggatg & & \\
\hline \multirow[t]{2}{*}{ Exon 18} & Intron 17 & Sense & gcatgggcagggtctgaaac & 3.5 & 284 \\
\hline & Intron 18 & Antisense & gctggcttcagggacaaagg & & \\
\hline \multirow[t]{2}{*}{ Exon 21} & Intron 20 & Sense & ggcttcatgtccaccttgtc & 2.0 & 256 \\
\hline & Intron 21 & Antisense & cggcaatgggtttctccttc & & \\
\hline \multirow[t]{2}{*}{ Exon 22} & Intron 21 & Sense & cccatttctactttgcetccc & 3.5 & 172 \\
\hline & Intron 22 & Antisense & tgggaaggcagccacctc & & \\
\hline \multirow[t]{2}{*}{ Exon 23} & Intron 22 & Sense & aaagggcatgtgctctgg & 2.0 & 378 \\
\hline & Intron 23 & Antisense & ccattgggaggaaggaagtc & & \\
\hline \multirow[t]{2}{*}{ Exon 24} & Intron 23 & Sense & gaagctcaagcgaggtacag & 2.0 & 229 \\
\hline & Intron 24 & Antisense & acgagatcttgccettgtgg & & \\
\hline Exon 26 & Intron 25 & Sense & ggttggtgccttctctttgc & 3.5 & 244 \\
\hline & Intron 26 & Antisense & ctcaggctgggctgaaagac & & \\
\hline Exon 27 & Intron 26 & Sense & gggatgagaggcagcaacag & 2.0 & 373 \\
\hline & Intron 27 & Antisense & gtccagctgacttgtatacc & & \\
\hline Exon 28 & Intron 27 & Sense & gacagaggtgccaccagtag & 3.5 & 450 \\
\hline (Part I) & Exon 28 & Antisense & cccgagagccattgctgttg & & \\
\hline Exon 28 & Exon 28 & Sense & acccatccaagatctcctac & 3.5 & 494 \\
\hline (Part II) & Exon 28 & Antisense & tggtggtgatgggctcgtag & & \\
\hline
\end{tabular}


Table III. Primers for PCR-SSP typing of SCN5A variants.

\begin{tabular}{|c|c|c|c|c|c|}
\hline $\begin{array}{l}\text { SCN5A } \\
\text { variation }\end{array}$ & $\begin{array}{l}\text { Gene } \\
\text { region }\end{array}$ & Direction & Specificity & Sequence $\left(5^{\prime}-3^{\prime}\right)$ & $\begin{array}{l}\text { Size } \\
\text { (bp) }\end{array}$ \\
\hline$-1062 \mathrm{~T}>\mathrm{C}$ & Promoter & $\begin{array}{l}\text { Sense } \\
\text { Sense } \\
\text { Antisense }\end{array}$ & $\begin{array}{l}1062 \mathrm{~T} \\
1062 \mathrm{C}\end{array}$ & $\begin{array}{l}\text { ccccataggtcctgggcat } \\
\text { ccccataggtcctgggcac } \\
\text { cagagcctggagtcacatac }\end{array}$ & $\begin{array}{l}127 \\
127\end{array}$ \\
\hline$-354 \mathrm{G}>\mathrm{C}$ & Promoter & $\begin{array}{l}\text { Sense } \\
\text { Sense } \\
\text { Antisense }\end{array}$ & $\begin{array}{l}-354 \mathrm{G} \\
-354 \mathrm{C}\end{array}$ & $\begin{array}{l}\text { cegcggacagacagacatag } \\
\text { gtatactctggcgggtgctgg } \\
\text { gtatactctggcgggtgctgc }\end{array}$ & $\begin{array}{l}138 \\
138\end{array}$ \\
\hline $287 \mathrm{C}>\mathrm{T}$ & Intron 1 & $\begin{array}{l}\text { Sense } \\
\text { Sense } \\
\text { Antisense }\end{array}$ & $\begin{array}{c}287 \mathrm{C} \\
287 \mathrm{~T} \\
\text { Generic }\end{array}$ & $\begin{array}{l}\text { gtccetgcgtgctcctcc } \\
\text { gtccctgcgtgctcctct } \\
\text { ggcgcacggtgtttagagac }\end{array}$ & $\begin{array}{l}145 \\
145\end{array}$ \\
\hline $87 \mathrm{G}>\mathrm{A}$ & Exon 2 & $\begin{array}{l}\text { Sense } \\
\text { Sense } \\
\text { Antisense }\end{array}$ & $\begin{array}{c}87 \mathrm{G} \\
87 \mathrm{~A} \\
\text { Generic }\end{array}$ & $\begin{array}{l}\text { catcgagaagcgcatggcg } \\
\text { catcgagaagcgcatggca } \\
\text { ggctctccgatgagctcttg }\end{array}$ & $\begin{array}{l}168 \\
168\end{array}$ \\
\hline $\begin{array}{l}1199 \mathrm{G}>\mathrm{C} \\
(\mathrm{G} 400 \mathrm{~A})\end{array}$ & Exon 10 & $\begin{array}{l}\text { Sense } \\
\text { Antisense } \\
\text { Antisense }\end{array}$ & $\begin{array}{l}\text { Generic } \\
1199 \mathrm{G} \\
1199 \mathrm{C}\end{array}$ & $\begin{array}{l}\text { cctggcacaactagactagg } \\
\text { gttcaccaggtagaaggacc } \\
\text { gttcaccaggtagaaggacg }\end{array}$ & $\begin{array}{l}166 \\
166\end{array}$ \\
\hline $\begin{array}{l}1673 \mathrm{~A}>\mathrm{G} \\
(\mathrm{H} 558 \mathrm{R})\end{array}$ & Exon 12 & $\begin{array}{l}\text { Sense } \\
\text { Sense } \\
\text { Antisense }\end{array}$ & $\begin{array}{l}1673 \mathrm{~A} \\
1673 \mathrm{G} \\
\text { Generic }\end{array}$ & $\begin{array}{l}\text { ggagagcgagagccacca } \\
\text { ggagagcgagagccaccg } \\
\text { ttgcagtccacagtgctgttc }\end{array}$ & $\begin{array}{l}139 \\
139\end{array}$ \\
\hline IVS16C $>\mathrm{T}$ & Intron 16 & $\begin{array}{l}\text { Sense } \\
\text { Sense } \\
\text { Antisense }\end{array}$ & $\begin{array}{c}\text { IVS16-6C } \\
\text { IVS16-6T } \\
\text { Generic }\end{array}$ & $\begin{array}{l}\text { gtgagcctgacccattatctc } \\
\text { gtgagcctgacccattatct } \\
\text { tggtggtgatgggctcgtag }\end{array}$ & $\begin{array}{l}299 \\
299\end{array}$ \\
\hline $\begin{array}{l}3578 \mathrm{G}>\mathrm{A} \\
(\mathrm{R} 1193 \mathrm{Q})\end{array}$ & Exon 20 & $\begin{array}{l}\text { Sense } \\
\text { Sense } \\
\text { Antisense }\end{array}$ & $\begin{array}{l}3578 \mathrm{G} \\
3578 \mathrm{~A} \\
\text { Generic }\end{array}$ & $\begin{array}{l}\text { agggaaggtctggtggcg } \\
\text { agggaaggtctggtggca } \\
\text { tcctgctctggcctccatac }\end{array}$ & $\begin{array}{l}304 \\
304\end{array}$ \\
\hline $\begin{array}{l}4786 \mathrm{~T}>\mathrm{A} \\
(\mathrm{F} 1596 \mathrm{I})\end{array}$ & Exon 27 & $\begin{array}{l}\text { Sense } \\
\text { Sense } \\
\text { Antisense }\end{array}$ & $\begin{array}{l}4786 \mathrm{~T} \\
4786 \mathrm{~A} \\
\text { Generic }\end{array}$ & $\begin{array}{l}\text { caacagctggaatatcttcgact } \\
\text { caacagctggaatatcttcgaca } \\
\text { gaagctagggttgtacatgg }\end{array}$ & $\begin{array}{l}148 \\
148\end{array}$ \\
\hline $5457 \mathrm{~T}>\mathrm{A}$ & Exon 28 & $\begin{array}{l}\text { Sense } \\
\text { Sense } \\
\text { Antisense }\end{array}$ & $\begin{array}{l}5457 \mathrm{~T} \\
5457 \mathrm{~A} \\
\text { Generic }\end{array}$ & $\begin{array}{l}\text { tcctgtctgactttgccgat } \\
\text { tcctgtctgactttgccgac } \\
\text { tcttcagggcgtccatctcc }\end{array}$ & $\begin{array}{l}180 \\
180\end{array}$ \\
\hline
\end{tabular}

PCR-SSP, polymerase chain reaction with sequence-specific primers.

In one $\mathrm{AMI} / \mathrm{VF}^{+}$patient (79 year old, male) the missense mutation 3578G $>$ A (R1193Q) was identified. The mutation was described as a normal variant with a frequency of $0.3 \%$ in Caucasians (32). However, the mutation conducts a longer QT-time and an association with LQTS has been discussed $(33,34)$. Associations have also been described with Brugada syndrome, progressive cardiac conduction defect (PCCD) and sudden infant death syndrome (30,34,35). Recently, the R1193Q variant was described in a young Korean patient (3 year old, male) with LQTS (36).

The missense mutation 4786T $>\mathrm{A}$ (F1596I) in exon 27 was found in one $\mathrm{AMI} / \mathrm{VF}^{+}$patient (54 year old, male). In a previous study the mutation was found in two out of 2,500 LQTS patients; however, not in the 1,300 individuals of the control group (37). An association with primary atrial fibrillation has been discussed; however, no evidence for an effect of the mutation on the channel function has been described (38).

In order to evaluate allele and genotype frequencies, the common SNPs $(87 \mathrm{G}>\mathrm{A}, 1673 \mathrm{~A}>\mathrm{G}$, IVS16-6C $>\mathrm{T}$ and $5457 \mathrm{~T}>\mathrm{A})$ were genotyped by $\mathrm{PCR}-\mathrm{SSP}$ in all patients (49 $\mathrm{AMI} / \mathrm{VF}^{+}$and $74 \mathrm{AMI} / \mathrm{VF}^{-}$) and controls (480 healthy blood donors). The differences in allele and genotype frequencies between the study groups were not identified to be statistically significant (Table V). The rare missense mutations $(3578 \mathrm{G}>\mathrm{A}$ and $4786 \mathrm{~T}>\mathrm{A})$ were screened by PCR-SSP in $\mathrm{AMI} / \mathrm{VF}^{-}$patients and controls. None of the AMI/VF74 patients were positive for the two mutations, whereas, one 
Table IV. Single nucleotide variants identified in the $S C N 5 A$ gene of $\mathrm{AMI} / \mathrm{VF}^{+}$patients.

\begin{tabular}{|c|c|c|c|c|}
\hline Gene region & Occurence $^{\mathrm{a}}$ & $\begin{array}{l}\text { Nucleotide } \\
\text { change }\end{array}$ & $\begin{array}{l}\text { Amino acid } \\
\text { change }\end{array}$ & $\mathrm{dbSNP}^{\mathrm{b}}$ \\
\hline Exon 2 & Common & $87 \mathrm{G}>\mathrm{A}$ & None & rs6599230 \\
\hline Exon $6 b$ & Rare & $630 \mathrm{G}>\mathrm{A}$ & None & rs193922727 \\
\hline Exon 12 & Common & $1673 A>G$ & His558Arg & rs1805124 \\
\hline Intron 16 & Common & IVS16-6C>T & Unknown & rs41260344 \\
\hline Exon 17 & Rare & $3183 \mathrm{G}>\mathrm{A}$ & None & rs7430407 \\
\hline Exon 20 & Rare & $3578 \mathrm{G}>\mathrm{A}$ & Arg1193Glu & rs41261344 \\
\hline Exon 26 & Rare & $4509 \mathrm{C}>\mathrm{T}$ & None & rs45548237 \\
\hline Exon 27 & Rare & $4786 \mathrm{~T}>\mathrm{A}$ & Phe1596Ile & rs199473278 \\
\hline Exon 28 & Common & $5457 \mathrm{~T}>\mathrm{A}$ & None & rs1805126 \\
\hline
\end{tabular}

${ }^{a}$ Each rare variant was found in only one of the 46 analyzed $\mathrm{AMI} / \mathrm{VF}^{+}$patients. AMI, acute myocardial infarction; VF, ventricular fibrillation; single nucleotide polymorphism database (dbSNP); ${ }^{\mathrm{d}} \mathrm{dbSNP}$ reference number.

Table V. Genotype frequency of SCN5A variants.

\begin{tabular}{|c|c|c|c|c|c|}
\hline Variant & Genotype $^{a}$ & $\begin{array}{c}\mathrm{AMI} / \mathrm{VF}^{+} \\
\mathrm{n}(\%)\end{array}$ & $\begin{array}{c}\mathrm{AMI} / \mathrm{VF}^{-} \\
\mathrm{n}(\%)\end{array}$ & $\begin{array}{c}\text { Controls } \\
\mathrm{n}(\%)\end{array}$ & $\begin{array}{l}\text { Significance } \\
\text { (P-value) }\end{array}$ \\
\hline$-1062 \mathrm{~T}>\mathrm{C}$ & TT & $49(100)$ & $74(100)$ & $480(100)$ & - \\
\hline$-354 \mathrm{G}>\mathrm{C}$ & GG & $49(100)$ & $74(100)$ & $480(100)$ & - \\
\hline $287 \mathrm{C}>\mathrm{T}$ & $\mathrm{CC}$ & 49 (100) & $74(100)$ & $480(100)$ & - \\
\hline \multirow[t]{3}{*}{$87 \mathrm{G}>\mathrm{A}$} & GG & $31(68.9)$ & $50(69.4)$ & $294(63.0)$ & $0.188^{b}$ \\
\hline & GA & $12(26.7)$ & $22(30.6)$ & $158(33.8)$ & $0.568^{c}$ \\
\hline & AA & $2(4.4)$ & $0(0)$ & $15(3.2)$ & $0.378^{\mathrm{d}}$ \\
\hline $1199 \mathrm{G}>\mathrm{C}$ & GG & 49 (100) & $74(100)$ & $480(100)$ & - \\
\hline \multirow[t]{3}{*}{$1673 \mathrm{~A}>\mathrm{G}$} & $\mathrm{AA}$ & $34(69.4)$ & $46(62.2)$ & $283(59.3)$ & $0.712^{\mathrm{b}}$ \\
\hline & $\mathrm{AG}$ & $14(28.6)$ & $26(35.1)$ & $168(35.2)$ & $0.345^{\mathrm{c}}$ \\
\hline & GG & $1(2.0)$ & $2(2.7)$ & $26(5.5)$ & $0.277^{\mathrm{d}}$ \\
\hline \multirow[t]{3}{*}{ IVS16-6C >T } & $\mathrm{CC}$ & $45(93.9)$ & - & $444(92.7)$ & - \\
\hline & $\mathrm{CT}$ & $3(6.1)$ & - & $34(7.1)$ & $0.919^{c}$ \\
\hline & $\mathrm{TT}$ & $0(0)$ & - & $1(0.2)$ & - \\
\hline \multirow[t]{3}{*}{$3578 \mathrm{G}>\mathrm{A}$} & GG & $48(98.0)$ & 74 (100) & 479 (99.8) & - \\
\hline & GA & $1(2.0)$ & $0(0)$ & $1(0.2)$ & $0.046^{\mathrm{c}}$ \\
\hline & AA & $0(0)$ & $0(0)$ & $0(0)$ & - \\
\hline \multirow[t]{3}{*}{$4786 \mathrm{~T}>\mathrm{A}$} & $\mathrm{TT}$ & $48(98.0)$ & $74(100)$ & $480(100)$ & - \\
\hline & TA & $1(2.0)$ & $0(0)$ & $0(0)$ & - \\
\hline & AA & $0(0)$ & $0(0)$ & $0(0)$ & - \\
\hline \multirow[t]{3}{*}{$5457 \mathrm{~T}>\mathrm{A}$} & $\mathrm{TT}$ & $20(42.6)$ & $30(44.8)$ & $208(44.3)$ & $0.669^{b}$ \\
\hline & TA & $20(42.6)$ & $32(47.8)$ & 209 (44.6) & $0.801^{\mathrm{c}}$ \\
\hline & $\mathrm{AA}$ & $7(14.8)$ & $5(7.4)$ & $52(11.1)$ & $0.973^{\mathrm{d}}$ \\
\hline
\end{tabular}

${ }^{\mathrm{a}}$ Genotypes: homozygous major allele, heterozygous, homozygous minor allele; ${ }^{\mathrm{b}} \mathrm{P} 1: \mathrm{AMI} / \mathrm{VF}^{+} \mathrm{vs}$. AMI/VF; ${ }^{\mathrm{c}} \mathrm{P} 2$ : $\mathrm{AMI} / \mathrm{VF}^{+}$vs. controls; ${ }^{\mathrm{d} P} 3$ : AMI vs. controls. AMI, acute myocardial infarction; VF, ventricular fibrillation.

of the 480 controls was positive for the $3578 \mathrm{G}>\mathrm{A}$ mutation. All study individuals were also screened for the SCN5A variants $(1062 \mathrm{~T}>\mathrm{C}, 354 \mathrm{G}>\mathrm{C}, 287 \mathrm{C}>\mathrm{T}$ and $1199 \mathrm{G}>\mathrm{C})$ that were associated with arrhythmia in previous studies $(21,24)$.
However, none of the patients or controls were positive for these variants.

In conclusion, mutations in the $S C N 5 A$ gene are relatively uncommon in $\mathrm{AMI} / \mathrm{VF}^{+}$patients. In the present study only 
two out of $49 \mathrm{AMI} / \mathrm{VF}^{+}$patients $(4.1 \%)$ demonstrated SCN5A variants that may be the cause of VF.

\section{Acknowledgements}

This study was supported by the DZHK (German Centre for Cardiovascular Research) and by the BMBF (German Ministry of Education and Research).

\section{References}

1. Zipes DP and Wellens HJ: Sudden cardiac death. Circulation 98: 2334-2351, 1998.

2. Spooner PM, Albert C, Benjamin EJ, et al: Sudden cardiac death, genes, and arrhythmogenesis: consideration of new population and mechanistic approaches from a National Heart, Lung, and Blood Institute workshop, Part II. Circulation 103: 2447-2452, 2001.

3. Zipes DP, Camm AJ, Borggrefe M, et al; European Heart Rhythm Association; Heart Rhythm Society; American College of Cardiology; American Heart Association Task Force; European Society of Cardiology Committee for Practice Guidelines: ACC/AHA/ESC 2006 guidelines for management of patients with ventricular arrhythmias and the prevention of sudden cardiac death: a report of the American College of Cardiology/American Heart Association Task Force and the European Society of Cardiology Committee for Practice Guidelines (Writing Committee to Develop Guidelines for Management of Patients With Ventricular Arrhythmias and the Prevention of Sudden Cardiac Death). J Am Coll Cardiol 48: e247-e346, 2006.

4. Friedlander Y, Siscovick DS, Weinmann S, et al: Family history as a risk factor for primary cardiac arrest. Circulation 97: 155-160, 1998.

5. Jouven X, Desnos M, Guerot C and Ducimetière P: Predicting sudden death in the population: the Paris Prospective Study I. Circulation 99: 1978-1983, 1999.

6. Kaikkonen KS, Kortelainen ML, Linna E and Huikuri HV: Family history and the risk of sudden cardiac death as a manifestation of an acute coronary event. Circulation 114: 1462-1467, 2006.

7. Novotny T, Kadlecova J, Raudenska M, et al: Mutation analysis ion channel genes ventricular fibrillation survivors with coronary artery disease. Pacing Clin Electrophysiol 34: 742-749, 2011.

8. Rook MB, Evers MM, Vos MA and Bierhuizen MF: Biology of cardiac sodium channel Nav1.5 expression. Cardiovasc Res 93 $12-23,2012$.

9. Schroeter A, Walzik S, Blechschmidt S, Haufe V, Benndorf K and Zimmer T: Structure and function of splice variants of the cardiac voltage-gated sodium channel $\mathrm{Na}(\mathrm{v}) 1.5$. J Mol Cell Cardiol 49: 16-24, 2010.

10. Tfelt-Hansen J, Winkel BG, Grunnet M and Jespersen T: Inherited cardiac diseases caused by mutations in the Nav1.5 sodium channel. J Cardiovasc Electrophysiol 21: 107-115, 2010.

11. Kapplinger JD, Tester DJ, Alders M, et al: An international compendium of mutations in the SCN5A-encoded cardiac sodium channel in patients referred for Brugada syndrome genetic testing. Heart Rhythm 7: 33-46, 2010

12. Remme CA, Wilde AA and Bezzina CR: Cardiac sodium channel overlap syndromes: different faces of SCN5A mutations. Trends Cardiovasc Med 18: 78-87, 2008.

13. Ruan Y, Liu N and Priori SG: Sodium channel mutations and arrhythmias. Nat Rev Cardiol 6: 337-348, 2009.

14. Wang DW, Desai RR, Crotti L, et al: Cardiac sodium channel dysfunction in sudden infant death syndrome. Circulation 115 368-376, 2007.

15. Watanabe H, Nogami A, Ohkubo K, et al: Electrocardiographic characteristics and SCN5A mutations in idiopathic ventricular fibrillation associated with early repolarization. Circ Arrhythm Electrophysiol 4: 874-881, 2011.

16. Park JK, Martin LJ, Zhang X, Jegga AG and Benson DW: Genetic variants in SCN5A promoter are associated with arrhythmia phenotype severity in patients with heterozygous loss-of-function mutation. Heart Rhythm 9: 1090-1096, 2012.

17. Marangoni S, Di Resta C, Rocchetti M, et al: A Brugada syndrome mutation (p.S216L) and its modulation by p.H558R polymorphism: standard and dynamic characterization. Cardiovasc Res 91: 606-616, 2011.
18. Cheng J, Tester DJ, Tan BH, et al: The common African American polymorphism SCN5A-S1103Y interacts with mutation SCN5A-R680H to increase late Na current. Physiol Genomics 43: 461-466, 2011

19. Shinlapawittayatorn K, Du XX, Liu H, Ficker E, Kaufman ES and Deschênes I: A common SCN5A polymorphism modulates the biophysical defects of SCN5A mutations. Heart Rhythm 8: 455-462, 2011.

20. Lahtinen AM, Noseworthy PA, Havulinna AS, et al: Common genetic variants associated with sudden cardiac death: the FinSCDgen study. PLoS One 7: e41675, 2012.

21. Hu D, Viskin S, Oliva A, et al: Novel mutation in the SCN5A gene associated with arrhythmic storm development during acute myocardial infarction. Heart Rhythm 4: 1072-1080, 2007.

22. Elmas E, Bugert P, Popp T, Lang S, Weiss C, Behnes M, Borggrefe $\mathrm{M}$ and Kälsch T: The P-selectin gene polymorphism Val168Met: a novel risk marker for the occurrence of primary ventricular fibrillation during acute myocardial infarction. J Cardiovasc Electrophysiol 21: 1260-1265, 2010.

23. Bugert P, Lese A, Meckies J, Zieger W, Eichler H and Klüter H: Optimized sensitivity of allele-specific PCR for prenatal typing of human platelet alloantigen single nucleotide polymorphisms. Biotechniques 35: 170-174, 2003.

24. Bezzina CR, Shimizu W, Yang P, et al: Common sodium channel promoter haplotype in asian subjects underlies variability in cardiac conduction. Circulation 113: 338-344, 2006.

25. Zhang T, Moss A, Cong P, et al: LQTS gene LOVD database. Hum Mutat 31: E1801-E1810, 2010.

26. Ackerman MJ, Siu BL, Sturner WQ, Tester DJ, Valdivia CR, Makielski JC and Towbin JA: Postmortem molecular analysis of SCN5A defects in sudden infant death syndrome. JAMA 286: 2264-2269, 2001

27. Takahata T, Yasui-Furukori N, Sasaki S, Igarashi T, Okumura K, Munakata A and Tateishi T: Nucleotide changes in the translated region of SCN5A from Japanese patients with Brugada syndrome and control subjects. Life Sci 72: 2391-2399, 2003.

28. Gouas L, Nicaud V, Berthet M, Forhan A, Tiret L, Balkau B and Guicheney P: Association of KCNQ1, KCNE1, KCNH2 and SCN5A polymorphisms with QTc interval length in a healthy population. Eur J Hum Genet 13: 1213-1222, 2005.

29. Lai LP, Su YN, Hsieh FJ, et al: Denaturing high-performance liquid chromatography screening of the long QT syndrome-related cardiac sodium and potassium channel genes and identification of novel mutations and single nucleotide polymorphisms. J Hum Genet 50: 490-496, 2005.

30. Arnestad M, Crotti L, Rognum TO, et al: Prevalence of long-QT syndrome gene variants in sudden infant death syndrome. Circulation 115: 361-367, 2007.

31. Zhang Y, Chang B, Hu S, et al: Single nucleotide polymorphisms and haplotype of four genes encoding cardiac ion channels in Chinese and their association with arrhythmia. Ann Noninvasive Electrocardiol 13: 180-190, 2008.

32. Ackerman MJ, Splawski I, Makielski JC, et al: Spectrum and prevalence of cardiac sodium channel variants among black, white, Asian, and Hispanic individuals: implications for arrhythmogenic susceptibility and Brugada/long QT syndrome genetic testing. Heart Rhythm 1: 600-607, 2004.

33. Wang Q, Chen S, Chen Q, et al: The common SCN5A mutation R1193Q causes LQTS-type electrophysiological alterations of the cardiac sodium channel. J Med Genet 41: e66, 2004.

34. Sun A, Xu L, Wang S, et al: SCN5A R1193Q polymorphism associated with progressive cardiac conduction defects and long QT syndrome in a Chinese family. J Med Genet 45: 127-128, 2008.

35. Huang H, Zhao J, Barrane FZ, Champagne J and Chahine M: Nav1.5/R1193Q polymorphism is associated with both long QT and Brugada syndromes. Can J Cardiol 22: 309-313, 2006.

36. Kwon HW, Lee SY, Kwon BS, et al: Long QT syndrome and dilated cardiomyopathy with SCN5A p.R1193Q polymorphism: cardioverter-defibrillator implantation at 27 months. Pacing Clin Electrophysiol 35: e243-e246, 2012.

37. Kapplinger JD, Tester DJ, Salisbury BA, et al: Spectrum and prevalence of mutations from the first 2,500 consecutive unrelated patients referred for the FAMILION long QT syndrome genetic test. Heart Rhythm 6: 1297-1303, 2009.

38. Olesen MS, Yuan L, Liang B, et al: High prevalence of long QT syndrome-associated SCN5A variants in patients with early-onset lone atrial fibrillation. Circ Cardiovasc Genet 5: 450-459, 2012. 